\title{
SHERLOCK HOLMES IN DER KULTURWISSENSCHAFT - EINE SPURENSUCHE MIT ERNST BLOCH
}

PD Dr. Francesca Vidal**

Sherlock Holmes zum Thema zu wählen, lässt den Verdacht aufkommen, dass hier jemand über heute als antiquiert geltende Figuren sprechen will, noch dazu um mit detektivischen Narrativen die Aufgabe von Kulturwissenschaft zu umreißen, als sei Kultur vergleichbar mit Kriminalfällen. Auffällig auch, dass es bei dem Rekurs auf Holmes hauptsächlich um Männer geht, auch dies wirkt ja ein wenig antiquiert, noch dazu um Männer, die H. C. Artmann beschreibt, als „Männer mit grauen flanellhüten, karierten sportmützen, dunklen melonen, deerstalkers, raffiniert geklebten falschbärten, künstlichen nasen, perücken, prima nachschlüsseln, revolverbelasteten hosenträgern, karbidlaternen, elektrischen stablampen, phantastisch vergrößernden lupen, stunden, ja tagelang lauernd, hinter schornsteinen, grabmälern, wartehäuschen, statuen, pissoirs, pfauengehegen, brückenpfeiler, sequoiabäumen, dachsbauten, laubenkolonien; als museumsbeamte verkleidet, als apachen, gaskasiere, großtanten, lebemännern, weinonkels, taxifahrer, als harmlose sonntagsjäger, aber dennoch stets auf der jagd nach dem gefährlichsten wild der welt - dem menschen in seinem wahn, der perfekteste verbrecher zu sein und nie gefaßt zu werden. Ich rede von den detektiven, wie sie uns schwarz auf weißen papier vors geistige auge treten ..."1

Soweit H. C. Artmann in seiner Beschreibung und tatsächlich will auch ich von diesen Detektiven reden, die so scheinbar nicht mehr in unsere Welt passen und vielleicht gerade deshalb Konjunktur haben, zumindest wenn man auf die neuesten Kriminalgeschichten schaut wie etwa den Roman, Handbuch für Detektive` von Jedediah Berry ${ }^{2}$.

Was mich an diesen Detektiven reizt, ist ihr auf der Jagd sein, auf der Jagd nach dem nur scheinbar Verborgenen, so wie eben auch ein Kulturwissenschaftler auf der Jagd ist nach dem nicht immer offen liegenden Teilen unserer Kultur. Und ist auch die Kultur kein Krimi, so ist sie doch ein Möglichkeitsraum, der auf den Menschen und

\footnotetext{
Universität Koblenz-Landau, Germany. Präsidentin der Ernst-Bloch-Gesellschaft.

${ }^{1}$ H. C. Artmann: Vorwort zu Detective Magazine der 13, hg. v. H. C. Artmann Esq., Salzburg: Residenz Verlag 1971.

2 Jedediah Berry: Handbuch für Detektive. München: Beck, 2010.
} 
seine Handlungen, damit immer auf Spuren verweist. Mit dem Philosophen Ernst Bloch richte ich den Blick auf diejenigen, die sich der Entschlüsselung scheinbarer Rätsel widmen. Dabei geht es mir nicht nur um seine Reflexionen detektivischer Methoden, sondern zugleich um die Frage, ob die Philosophie von Bloch als akribische Spurensuche gelesen werden kann. Ziel ist es, herauszufinden, ob in beidem Hinweise enthalten sind für die Jagd nach Erkenntnis, nachgerade für eine Orientierungskunst in der Wissenschaft. Dabei will ich den Menschen nicht per se auf den Verbrecher in der Unterwelt reduzieren, gehe aber vom Gestalter aus, der gemeinsam mit anderen die Lebenswirklichkeit formt und Kultur schafft und dem Oskar Negt nachsagt, ihm komme durch das Schwinden des Politischen und durch die immer schwierige Grenzziehung zwischen Privat und Öffentlich, also zwischen Nähe und Distanz, die Kultur abhanden. Dieses Schwinden aber muss - folgt man dem Denken des Detektivs - Spuren hinterlassen, die es zu suchen gilt, wenn man erkennen will, was es damit auf sich hat. Oder dialektisch gesprochen, wer das Wahre der Kultur enthüllen will, also davon ausgeht, dass sie etwas Widerständiges und Antizipatorisches enthalten muss, der muss die Spuren suchen, die den Widerspruch zur Realität aufzeigen.

Dieser Kulturbegriff geht durchaus auf die ursprüngliche Bedeutung zurück, so wie sie erstmalig von Cicero in Gesprächen auf seinem Landsitz in Tusculum entwickelt wurde, mithin auf die Pflege der Seele. ${ }^{3}$ Für Cicero benötigt die menschliche Seele Pflege, wenn eine civitas zum Wohle aller entwickelt werden soll, denn so wie Natur bearbeitet werden muss, um zum fruchtbaren Acker zu werden, bedarf die Entwicklung des Menschen und seiner Welt der Pflege, damit der Bildung. Kultur ist somit Ergebnis eines Prozesses, Pflege der Seele aber ist für Cicero die Philosophie: Cultura autem animi philosophia est. Als Aufgabe lässt sich dann der Prozess der Humanisierung ableiten, als Arbeit am Unbehagen an der Kultur die Frage, was dem entgegensteht. Insofern hätte auch ich gerne ein Handbuch für Detektive, im Sinne der Bedeutung von ,to detect ${ }^{\star}$ aufdecken. Aufdecken will ich, wie Kultur - mit Hamid Reza Yousefi - verstanden als „offenes Netzwerk von Perspektiven und dynamischen Prozessen; [...] das Ausdruck menschlicher Produkte ist, die sich gegenseitig

\footnotetext{
${ }^{3}$ Vgl. Burkhard Dücker: Kultur, in: Historisches Wörterbuch der Rhetorik. Hg. v. Gert Ueding. Tübingen 1998, Bd. 4, Sp. 1384-1420.
}

\begin{tabular}{|l|l|l|l|l|}
\hline Revista Dialectus & Ano 1 & n. 2 & Janeiro-Junho 2013 & p. 95-111 \\
\hline
\end{tabular}


beeinflussen und verändern“4 zum Sinn- und Orientierungssystem überhaupt werden kann.

Hierfür suche ich eine Methode der Spurensuche und ich finde mich da in guter Gesellschaft, hat sich doch bekannter Weise schon Sigmund Freud und mit ihm die Psychoanalyse an den Methoden des Detektivs orientiert, nicht nur in Bezug auf das Individuelle, sondern vor allem um dem Unbehagen gegenüber der Kultur begegnen zu können. Sein Motto für die Traumdeutung entnahm er Vergil. Es lautete: Flectere si nequeo Superos, Acheronta moveba (Kann ich die höheren Mächte nicht beugen, will ich doch die Unterwelt bewegen) und es wird meist in dem Sinn interpretiert, dass der verborgene Teil der Realität nicht weniger wichtig für die Entschlüsselung derselben sei als der sichtbare. ${ }^{5}$ Ginzburg und andere haben gezeigt, dass Freuds narrative Methode mit der detektivischen Arbeit von Holmes zu vergleichen ist und Freud sich dessen auch bewusst war, wenn er Handeln der Gegenwart erklären wollte, indem er vergangene Ereignisse zu rekonstruieren versuchte und die Spuren dieser Vergangenheit deshalb deutete.

Um jedoch die Analogie zum Detektiv zu verstehen, greife ich noch einmal auf den Vergleich des Detektivs mit dem Jäger an, womit H. C. Artmann im Grunde sagt, dass die Methoden des Detektivs älter sind als sein Beruf und noch dazu aus der Alltagspraxis kommen. Auch Carlo Ginzburg ${ }^{6}$, der sich mit der Freudschen Nähe zum Detektiv beschäftigt, verweist auf die Jäger, die wohl als erste die Kenntnisse der Spurensuche nutzen, um das Wild zu erlegen. Sein ,Indizienparadigma‘ ruft dazu auf, sich an den oft unbemerkt bleibenden Details zu orientieren, um durch Spurensicherung zur Kontextualisierung zu gelangen. Er erinnert an den Kunstkritiker Giovanni Morelli, der alte Meister bestimmen wollte und sich dabei an scheinbar Abwegigen orientierte wie die Darstellung von Ohren oder Fingerkuppen. Der Morelli Methode aber bediente sich auch Freud, er erkannte die Verwandtschaft der Verfahren, aus der Deutung der oft nicht beachteten Kleinigkeiten zur Erkenntnis zu gelangen.

Mit dem Hinweis auf die kleinsten Kleinigkeiten sind wir schon fast bei Ernst Bloch, der sich in seiner Enzyklopädie der Hoffnung auf das konzentriert, was denen

\footnotetext{
${ }^{4}$ Hamid Reza Yousefi: Phänomenologie des Eigenen und des Fremden. Eine interkulturelle Perspektive, in: Hamid Reza Yousefi, Klaus Fischer, Regine Kather, Peter Gerdsen, Hg.: Wege zur Kultur. Gemeinsamkeiten - Differenzen - Interdisziplinäre Dimensionen. Nordhausen 2008, S. 25-52, hier S. 29. ${ }^{5}$ Vgl. z. B. Carlo Ginzburg. „Indizien: Morelli, Freud und Sherlock Holmes“. In: Umberto Eco; Thomas A. Sebeok, Hg. Der Zirkel oder im Zeichen der Drei. Dupin, Holmes, Peirce. München 1985. 125 -179.

${ }^{6}$ Carlo Ginzburg. „Indizien: Morelli, Freud und Sherlock Holmes“. In: Umberto Eco; Thomas A. Sebeok, Hg. Der Zirkel oder im Zeichen der Drei. Dupin, Holmes, Peirce. München 1985. 125 -179.
}

\begin{tabular}{|l|l|l|l|l|} 
Revista Dialectus & Ano 1 & n. 2 & Janeiro-Junho 2013 & p. 95-111 \\
\hline
\end{tabular}


entgeht, die ihren Blick nicht genügend auf die Kleinigkeiten richten, auf die Dinge und Gegebenheiten, die im Nebenbei wirken und gerade deshalb zum Fragen auffordern und dabei zugleich auf die Antwort weisen. Deshalb orientiert er sich, wie andere Philosophen auch, wenn es um die Figur des Spurensuchers geht, nicht an Jägern, an Morelli oder Freud, sondern an der Schilderung desjenigen, der entschlüsselt, indem er Indizien im Verborgenen sucht und damit Bewegung in das Geschehen bringt, am Detektiv also, so wie ihn der Leser der ersten Kriminalgeschichten kennen gelernt hat. ${ }^{7}$ Dabei ist es gerade die Deutung der Indizien, die als durchgängiges Element aller Detektivromane bis heute augenfällig ist, selbst wenn sich die Methoden im Laufe der Zeit wandeln und selbst wenn es wie mit Handke, Dürrenmatt, Robbe-Grillet oder Eco beginnend immer mehr um die labyrinthische und schwer durchschaubare Wirklichkeit geht. Und so heißt es auch im jüngst erschienenen ,Handbuch für Detektive“: „Auch Dinge haben ein Gedächtnis. Der Türknauf erinnert sich an denjenigen, der ihn gedreht, das Telefon an den, der den Hörer abgenommen hat. Das Gewehr weiß noch, wann es das letzte Mal abgefeuert wurde, und vom wem. Aufgabe des Detektivs ist es, die

Sprache dieser Dinge zu lernen, damit er sie verstehen kann, wenn sie ihm etwas mitzuteilen haben. ${ }^{\text {"8 }}$ Es ist dies als erstes ein Denken vom Objekt her, objektive

Hermeneutik wenn man so will, aber eines das dann doch auf das Subjekt als Leser der Zeichen setzt, im Sinne der Bedeutung von Methode als mit dem Weg der Sache gehen.

Dieses Achten auf die kleinste Kleinigkeit kennzeichnet demnach jeden Spurensucher, der nicht an scheinbaren Tatsachen festhält, sondern an den Widersprüchen in den Dingen selbst. Es sind die Widersprüche, die dazu aufrufen, dem Selbstverständlichen zu misstrauen. Insofern lassen Spuren das fragende Staunen zum Anlass der Reflexion geraten. Immer wieder macht der Detektiv die Erfahrung, dass die Dinge nicht so sind wie sie scheinen. Deshalb erklärt Sherlock Holmes seinem Freund und Helfer Dr. Watson sehr oft: „Nichts ist trügerischer als eine offensichtliche Tatsache.“ (So z. B. in: Das Rätsel von Boscombe Valley) und Ernst Bloch erklärt die Schwierigkeiten im Prozess des Erkennens im 13ten Kapitel des Experimentum Mundi mit ähnlichen Worten: „Nichts schien einfacher zu sein, als die Dinge zu nehmen, wie

\footnotetext{
${ }^{7}$ Vgl. Ernst Bloch. Philosophische Ansicht des Detektivromans. In: Ders.: Literarische Aufsätze. Frankfurt am Main 1984. 242-263. Bei diesem Aufsatz handelt es sich um eine veränderte Fassung eines in Tübingen gehaltenen Vortrags, der erstmals in der Neuen Rundschau 71, 1960, 665-683 abgedruckt wurde.

${ }^{8}$ A.a.O. S. 22.
}

\begin{tabular}{|l|l|l|l|l|}
\hline Revista Dialectus & Ano 1 & n. 2 & Janeiro-Junho 2013 & p. 95-111 \\
\hline
\end{tabular}


sie sind. Sie sind aber nicht, als was sie uns erscheinen, das Denken macht es uns gerade mit dem erscheinend Seienden sauer. ${ }^{\text {} 9}$

Gerade jedoch weil es so schwierig ist, fordert der Erkenntnisprozess ein Einlassen auf Indizien, die es dem Menschen ermöglichen, Schlüsse und Mutmaßungen $\mathrm{zu}$ ziehen. Die Spuren werden gelesen, um sie dann in den richtigen Bedeutungszusammenhang stellen zu können. Dazu braucht ein Detektiv Kenntnisse, aber vor allem auch die Beobachtungsgabe und Kombinationsgeschick. Erst diese drei Talente zusammen, charakterisieren eine bestimmte Art von Detektiv. Gemeint ist also nicht der, der sein Ergebnis notfalls mit Gewalt heraus prügelt, der ist eine Figur des Thrillers der zwanziger und dreißiger Jahre des letzten Jahrhunderts, der sog. Hardboiled-school. Bei Dashiell Hammett oder Raymond Chandler wird sogar jeder Dialog zur sprachlichen Prügelei, der Detektiv führt Rededuelle, weshalb die Sprache seine Waffe ist, sowohl um den Gegner einzuschüchtern als auch um sich selbst zu behaupten. Unser Detektiv ist ein Faktensammler und kombinatorischer Rätselrater. Jemand wie Sherlock Holmes, auch oder gerade weil dieser gegenüber Watson behauptet, er rate nie, denn das sei ,eine entsetzliche Angewohnheit - zerstöre das logische Denkvermögen“, wogegen er der Wissenschaft der Deduktion folgen würde. ${ }^{10}$

Dass dem nicht ganz so ist, haben u. a. Umberto Eco und Thomas A. Sebeok gezeigt. ${ }^{11}$ Und nur weil es nicht ganz so ist, lassen sich dann auch Detektiv und Philosoph vergleichen.

Aber bleiben wir erst bei der Frage, auf welche Art Detektiv sich denn nun der Philosoph Bloch bezieht. Hier finden sich viele Ambivalenzen zu Denkern seiner Zeit, mit denen Bloch im Dialog stand wie etwa Walter Benjamin, Bert Brecht oder Siegfried Kracauer. Alle sprechen vom Detektiv, bevorzugen einen bestimmten Typus und beziehen sich grundsätzlich auf den Detektiv der Literatur Ihrer Zeit. Es ist ihr Musterdetektiv, der an der Figur des Sherlock Holmes, veranschaulicht wird: einer der raue Gewalt als Methode der Wahrheitsfindung ablehnt, kein Sam Spade also, sondern einer der sowohl aus der Ermittlung der Fakten als auch durch seine Fähigkeiten der Kombinatorik und Imagination das zunächst Verworrene einer vernünftigen Lösung

\footnotetext{
${ }^{9}$ Ernst Bloch. Experimentum Mundi. Frankfurt am Main: Suhrkamp, 1975. S. 60.

${ }^{10}$ So äußert sich Sherlock Holmes gegenüber Watson in dem Kapitel 'Die Wissenschaft der Deduktion' in einem Gespräch über einem französischen Detektiv namens François le Villard in ,Eine Studie in Scharlachrot', vgl. hierzu Thomas A. Seboek, Jean Umiter-Seboek: ,Sie kennen ja meine Methode“ Charles S. Peirce und Sherlock Holmes, in: Jochen Vogt, Hg.: Der Kriminalroman. Poetik - Theorie - Geschichte. München: Fink, 1998, S. 297-322.

${ }^{11}$ Vgl. Umberto Eco; Thomas A. Sebeok, Hg. Der Zirkel oder im Zeichen der Drei. Dupin, Holmes, Peirce. München 1985.
}

\begin{tabular}{|l|l|l|l|l|}
\hline Revista Dialectus & Ano 1 & n. 2 & Janeiro-Junho 2013 & p. 95-111 \\
\hline
\end{tabular}


nahe bringt. Methodisch orientiert sich ein solcher Detektiv immer an den Gesetzen der Logik und - dies aber viel versteckter - an den Regeln der Rhetorik, denn überzeugt wird der Leser gerade dadurch, dass er in den Prozess des Findens involviert wird. Insofern ist der Erfolg der am Ende präsentierten Lösung immer auch Ergebnis eines persuasiven Aktes.

Selbstverständlich entsprechen die Methoden der Detektive der jeweiligen Zeit, so geht ein Holmes naturwissenschaftlich-induktiv vor, ein Hercule Poirot eher intuitiv, in dem er sich auf die synaptischen Verknüpfungen ,seiner kleinen grauen Zellen' verlässt. Immer aber sind sie Spurensucher, denen ein ganz bestimmter Habitus eigen ist. Für Benjamin zeigt sich dieser detektivische Charakter im physiologischen Blick des Flaneurs $^{12}$, für Kracauer, der dem Detektivroman ein philosophisches Traktat widmete, wird diese Figur des Detektivs zur ratio selbst. Er schreibt: „Der Detektiv schweift in dem Leerraum zwischen den Figuren als entspannter Darsteller der ratio, die sich mit dem Illegalen auseinandersetzt, um es, gleich dem Sachverhalten des legalen Betriebs, zu dem Nichts ihrer eigenen Indifferenz zu zerstäuben. Er richtet sich nicht auf die ratio, sondern ist ihre Personifikation, er erfüllt nicht als Kreatur das von ihr Geheißene, sie selber vielmehr vollstreckt ihren Auftrag in seiner Unperson - [...]. Als den Figuranten der ratio, dessen Rolle nicht wechselt, entlarvt ihn die Tatsache zumal, dass er von jedem Autor einen Namen empfängt, der ihm zum unveräußerlichen Kennwort wird. Heiße er Sherlock Holmes, Roulettabille, Joe Jenkins: die Bezeichnung bleibt ihm erhalten in der Erscheinungen Flucht, und so bleibt auch er selber der gleiche. “13

Egal ob der Jäger, oder wie bei Kracauer der Arzt, oder wie bei Poe der Kartenspieler als Beispiel zur Beschreibung der Methode herangezogen werden, immer geht es darum, die detektivische Methode als das Entziffern von Zeichen zu kennzeichnen. Kracauer nimmt das Bild des Arztes, da der Detektiv zwar nicht heilen will, aber „die Krankheit am Gesellschaftsleib“14 zum Anlass der Deduktion nimmt, um dann das Geheimnis aus den Indizien zu entwirren. Freilich verrät uns dies, dass die Tat hier noch als Abweichung gesehen wird, zudem ist sie schon begangen, das intellektuelle Bemühen des Detektivs sucht Aufklärung des Vergangenen.

\footnotetext{
${ }^{12}$ Walter Benjamin: Kriminalromane, auf Reisen (1930), in: Jochen Vogt, Hg.: Der Kriminalroman. Poetik - Theorie - Geschichte. München: Fink, 1998, S. 23-24.

${ }^{13}$ Siegfried Kracauer. Der Detektiv-Roman. Ein philosophischer Traktat. Frankfurt am Main: Suhrkamp, 1979, S. 51-53

${ }^{14}$ Ebd. S. 63.
}

\begin{tabular}{|l|l|l|l|l|} 
Revista Dialectus & Ano 1 & n. 2 & Janeiro-Junho 2013 & p. 95-111 \\
\hline
\end{tabular}


Auch Edgar Allan Poe, dem man nachsagt, seine im April 1841 erschienene Erzählung, The Murders in The Rue Morgue' ${ }^{15}$ hätte die Detektivgeschichte ins Leben gerufen, kennzeichnet das ,Wie' des Schlussfolgerns als fortgesetztes Bemühen um Vernunftschlüsse. Es sei dies eine Geisteshaltung des Erzählers, die Poe mit dem Begriff der ,ratiocination' benennt. In „Die Morde in der Rue Morgue“ beschreibt er, wie Nancy Harrowitz herausgearbeitet hat, die gesamte Skala möglicher Erkenntnismethoden eines Detektivs am Beispiel des Kartenspielers, jemand also, der immer auch auf das Glück setzt und vor allem Spieler ist: „Ableitungen, Rückwärtsdenken, visuelle, sensorische und auditive Zeichen, das Lesen von Gesichtsausdrücken“ etc. kennzeichnen sein Vorgehen. ${ }^{16}$ Dieses ist durch philosophisches Denken geschult, weshalb es der polizeilichen Ermittlungsarbeit gegenüber überlegen ist, wie Gabriela Holzmann nicht umsonst im Hist. Wörterbuch der Rhetorik darlegt: „Ausgelöst durch eine falsche Fährte vertreten diese (die Polizisten) zunächst eine allgemein akzeptierte These (locus oder opinio communis) über die strittige Bedeutung von Spuren, die dann die Antithese (antitheton) des

Detektivs provoziert und die scharfsinnige Beweisführung eröffnet."17 Unter rhetorischen Gesichtspunkten ist auffällig wie sehr eine scharfsinnige Beweisführung dem deduktiven Verfahren der Argumentation folgt. Diese Fähigkeiten werden alle an Poes Gestalt Dupin orientierten Detektive haben, heißen sie nun Sherlock Holmes, Monsieur Poirot, Lord Peter Wimsey, Wachtmeister Studer oder anders. Ihre Scharfsinnigkeit lässt den Leser oft darüber hinweg sehen, dass die Deduktion so selbstverständlich und eindeutig nicht ist. Nancy Harrowitz vergleicht wie auch Eco, Sebeok und Ginzburg deren detektivische Methode mit den Theorien des amerikanischen Pragmatikers Charles Sanders Peirce, um zu belegen, dass die Detektive sich der Abduktion bedienen. Sie lesen Indizien, die andere unbeachtet ließen und wählen dann die am naheliegendste Hypothese. Das gelingt, weil ihre Spurensuche immer auch ein intuitives Spurensammeln ist, und ihnen im Lauf der Gattungsgeschichte, spätestens aber bei Agatha Christie der Zufall zur Hilfe kommt.

Führt man sich diese Detektive vor Augen wird eine Parallele erkennbar mit denen, die sich in intellektuellen Schaffensprozessen befinden, so wie Ernst Bloch dies

\footnotetext{
${ }^{15}$ Erstmals erschienen in Graham's Magazine, Volume 18, Philadelphia, 1841.

${ }^{16}$ Nancy Harrowitz. „Das Wesen des Detektiv Modells. Charles S. Peirce und Edgar Allan Poe“, in: Umberto Eco, Thomas A. Sebeok, Hg.: Der Zirkel oder Im Zeichen der Drei. Dupin, Holmes, Peirce. München 1985, S. 262-287, hier S. 273.

${ }^{17}$ Gabriela Holzmann: Kriminalliteratur, In: Historisches Wörterbuch der Rhetorik. Hg. v. Gert Ueding. Bd. 4. Tübingen 1998, Sp. 1377 - 1398, hier Spalte 1379.
}

\begin{tabular}{|l|l|l|l|l|} 
Revista Dialectus & Ano 1 & n. 2 & Janeiro-Junho 2013 & p. 95-111 \\
\hline
\end{tabular}


anhand seines Verständnisses von ,Jugend, Zeitwende und Produktivität' schildert. ${ }^{18}$ In dieser Beschreibung wird der Detektiv zur Projektionsfläche für den wissenschaftlich Tätigen. Bloch erklärt uns, dass die produktiv-tätige Subjektivität an die gesellschaftlichhistorischen Bedingungen gebunden ist, die sich an den Spuren in der Welt festmachen lassen. Auch er betont die Bedeutung der Intuition, sein Subjekt wittert Morgenluft. Sein Anspruch ist der eines Detektivs: allerdings auf Zukunft hin gerichtet, das ist der entscheidende Unterschied. Das Denken beginnt quasi nach dem Lesen der Spuren. Ziel ist es, jenseits der Erscheinungen das Wesentliche aufzuspüren und das Wahre zu ergründen und beides voranzutreiben. In der Phase der Inkubation befindet sich das Subjekt im Zustand des Meinens und Spekulierens. Es zielt auf das Gesuchte, deshalb ist es sensibel für die Spuren. Dem folgt eine meist blitzhafte Klärung, die, da sie als übermächtig erscheint, mit dem Terminus Inspiration bezeichnet wird, ohne damit transzendenter Mystik das Wort zu geben. Das Zündende der Inspiration liegt im Zusammentreffen der schöpferischen Anlage des tätigen Subjekts und der objektiven Gegebenheiten. Deshalb sagt Bloch in Prinzip Hoffnung: „Die

Inspiration insgesamt kommt derart, wann immer sie eine werkbildende ist, aus der Zusammenkunft von Subjekt und Objekt, aus der Zusammenkunft ihrer Tendenz mit der objektiven Tendenz der Zeit, und ist der Blitz, womit diese Konkordanz anhebt.“19 Explikation als Akt der Ausarbeitung ist so gesehen das Erzählen der Geschichte, die sich aus dem Lesen der Spuren ergibt. Beim Detektiv beleuchtet sie das Vergangene, beim Philosophen weist sie in die Zukunft.

In Blochs Ausführungen über den Detektivroman betont er, dass das Genre durch Poe entwickelt und zum Muster für die dann folgenden Geschichten wurde. Das Auftauchen dieser Art des Detektivs und dessen auf Vernunft gründende Methode erklärt er mit dem Aufkommen der Erkenntnisse der Aufklärung. Während vor der Mitte des 18. Jahrhunderts das Geständnis, also die Folter als Instrument der Wahrheitsfindung diente, fordert erst das mit der Aufklärung aufkommende Gebot der Menschlichkeit Indizien und damit entsprechende detektivische Methoden. So lesen wir bei Bloch: „Weil erst das Indizienverfahren zureichende Hinweise verlangt, bereits für einen Haftbefehl, gar erst vor Gericht, und damit zum kriminalistisch Aufdeckenden, mit dem Detektiv im Vordergrund, erst den Auftrag gab. Zeichen jeder Art,

\footnotetext{
${ }^{18}$ Ernst Bloch: Das Prinzip Hoffnung. Frankfurt am Main 1985, S. 132ff. Vgl. hierzu Francesca Vidal: Kunst als Vermittlung von Welterfahrung. Zur Rekonstruktion der Ästhetik von Ernst Bloch. Würzburg 1994, S. 49-54.

${ }^{19}$ Ernst Bloch: Das Prinzip Hoffnung, a.a.O. S. 141
}

\begin{tabular}{|l|l|l|l|l|} 
Revista Dialectus & Ano 1 & n. 2 & Janeiro-Junho 2013 & p. 95-111 \\
\hline
\end{tabular}


Bodenspuren, unechte Alibis und die Schlüsse aus allem sind nun so wichtig geworden wie selbst das alte, oft viel zu breite Cui bono.“20

Nur bleibt das Interesse an der Kriminalhistorie eher ein soziologisches, was den Philosophen und mit ihm viele Leser am Detektiv begeistert, ist das Vergnügen an den Formen seiner literarischen Gestaltung. Die Detektivgeschichte gewinnt von Berichten über Verbrechen, Schauerroman und Kolportage, schafft aber als neues Spannung erzeugendes Element den Detektiv, mit dessen Figur sich die Kennzeichen der Geschichte entfalten. Diese sind Bloch zufolge ,dreifach, hängen eng zusammen, sind des Abgezielten voll. Da ist zuerst die Spannung des Ratens; sie weist als ohnehin detektivisch, zum zweiten auf das Entlarvende, Aufdeckende hin, mit dem besonderen Akzent des Abseitigen, woraus oft das Wichtigste zu erfahren ist; und das Aufdeckende geht zum dritten auf Vorgänge, die aus ihrem Unerzählten, Vor-Geschichtehaften erst herauszubringen sind. Dies dritte Kennzeichen ist das charakteristische der Detektivgeschichte und macht sie, sogar weit vom Detektiv, unverwechselbar. Vor ihrem ersten Wort, vor dem ersten Kapitel geschah etwas, niemand weiß es, scheinbar auch der Erzähler nicht. Ein dunkler Punkt ist also noch unerkannter da, von dem her und zu dem hin sich die ganze Wagenladung der folgenden Ereignisse in Bewegung setzt, eine Untat, meist eine mörderische, steht vor Anfang. In allen anderen Erzählformen entwickeln sich die Taten wie Untaten vor einem durchaus anwesenden Leser, hier dagegen ist er bei einer Untat, einer den Tag scheuenden, obzwar besonders fertig ins Haus gelieferten, nicht dabei gewesen, sie liegt im Rücken der Geschichte, muß ans Licht gebracht werden, und dies Herausbringende ist selber und allein das Thema. Das dunkel Geschehene wird auch in keiner Vorhandlung dargestellt, eben weil es überhaupt noch nicht darstellbar ist, außer durch Ausgrabung, durch Indizien, welche rekonstruieren lassen.“21

Das Verbrechen ist sowohl Anlass als auch Movens der Geschichte, sein Ungelöstsein weckt die Aufmerksamkeit, richtet das Interesse des Lesers auf die Leistung des Detektivs, weil er die im Verborgenen liegende Geschichte rekonstruieren will. So folgt der Leser dem Detektiv wie der Hörende einem Redner, da er zur Lösung gelangen will. Für Bloch sind es daher nicht immer die schlechtesten Leser, die schon einmal auf das Ende schauen. Die erste Geschichte ist die, die der Verbrecher

\footnotetext{
${ }^{20}$ Ernst Bloch. Philosophische Ansicht des Detektivromans. In: Ders.: Literarische Aufsätze. Frankfurt am Main 1984; S. 243f.

${ }^{21}$ Ebd. S. 247.
}

\begin{tabular}{|l|l|l|l|l|}
\hline Revista Dialectus & Ano 1 & n. 2 & Janeiro-Junho 2013 & p. 95-111 \\
\hline
\end{tabular}


konstruiert hat und die verborgen ist, weil dieser versucht hat, die Spuren zu beseitigen. Aber einige Zeichen sind seiner Aufmerksamkeit entgangen und werden vom Detektiv in ihrer Bedeutung gelesen. Er muss die Welt deshalb als eine Sammlung von Spuren sehen, deren Polyvalenz er dadurch reduziert, dass er die Bedeutung der Spuren erkennt. Der Leser verfolgt sein Tun, nimmt Teil an der Rekonstruktion der Geschichte des Verbrechers, die ihm dann in der Lösung des Falls präsentiert wird. Deshalb ist es nicht der durch eine veränderte Rechtssprechung notwendig gewordene Detektiv im Staatsdienst, der die Neugier Blochs weckt, sondern Erzählungen, die das Wie des Findens zum Spannungselement haben. Eine Form des Geschichtenerzählens, die wie Bert Brecht darlegt ${ }^{22}$, so großen Genuss bereite, da der Leser motiviert wird, seine Vorurteile zu überwinden und zu vernünftigen Urteilen $\mathrm{zu}$ gelangen. Den strengsten Kodex habe der englische Roman, der sei besonders fair, täusche den Leser nicht, sondern unterbreite alles Material, so dass er die Lösung selbst in Angriff nehmen kann. Lust bereite die Denkaufgabe, also das Beobachtungen anstellen und das Schlüsse ziehen, um dann vor allem zu Entschlüssen zu kommen. Während der Mensch seine

Beobachtungen im Alltag immer seltener verwerten könne, er hier weder Herr über Schlüsse noch Entschlüsse sei, würde in der Welt des Romans das Kausalitätsgesetz funktionieren. Hier entspreche das Wahrscheinlichste tatsächlich der Lösung. Wobei angemerkt sei, dass Brecht nicht darauf eingeht, dass die aristotelische Formel im Grunde umgekehrt wird, denn das Verbrechen soll als wahr, nicht aber als wahrscheinlich gelten. Gerade in den ersten Kriminalromanen ist es noch eine Ausnahme, sein Geschehen ist eher unwahrscheinlich, aber eben dennoch geschehen. Viel später hat selbst das Verbrechen Regeln und System, was dann neue Varianten des Ermittlers hervorruft.

Trotzdem: Die Detektivgeschichte lehrt das Denken. Der Leser sammelt während des Lesens wie der Held der Geschichte Spuren, auch er muss sie ordnen und in den Bedeutungszusammenhang stellen, damit er die Möglichkeit hat, die vom Detektiv präsentierte Lösung auch als logische nachzuvollziehen, was immer auch heißt, dass sich die Logik auch aus der Form der Präsentation ergibt. Der Detektiv arbeitet daher auch wie ein Redner, hat beispielsweise ähnliche Suchformeln, wie die, die ein Redner in der Phase der Inventio nutzt: Quis, Quid, Ubi, Quibus auxiliis, Quomodo, Quando - Wann, Wo, Welche Mittel, Wer hatte Zugang, Wer ist der Tote,

\footnotetext{
${ }^{22}$ Vgl. Bertolt Brecht. „Über die Popularität des Kriminalromans“. Schriften zur Literatur und Kunst 2, Gesammelte Werke 19. Frankfurt am Main: 1969. 450 - 457.
}

\begin{tabular}{|l|l|l|l|l|} 
Revista Dialectus & Ano 1 & n. 2 & Janeiro-Junho 2013 & p. 95-111 \\
\hline
\end{tabular}


War es ein Mord, damit werden die Zeugen konfrontiert, deren Glaubwürdigkeit im Gegensatz zu der des Detektivs in Zweifel steht. ${ }^{23}$

Wenn auch die Welt undurchschaubar erscheint, wird durch das Vergnügen an der greifenden Logik des Romans zugleich die Freude an einer Erkenntnisleistung vermittelt, die in praktisches Tun übergeht. Der Geschichte gelingt es also, das Verfahren des Detektivs vergnüglich als Weg zur Erkenntnis zu präsentieren. Und so ist das Ende wie der Schluss der Gerichtsrede: zusammenfassende Rekonstruktion und Entlarvung.

In seiner Analyse der Detektivgeschichte arbeitet Bloch heraus, dass dieses Verfahren seine Bedeutung gerade in einer durch Entfremdung geprägten Gesellschaft erhält. Der Roman bringe den Zustand der Entfremdung auf vergnügliche Weise zum Ausdruck und zeige, dass die Spurensuche in der entfremdeten Welt zum Weg des Zurechtfindens wird. Gerade die Darstellung der entfremdeten Welt, einer in der der Mensch sich selbst und dem anderen gegenüber fremd geworden ist, verbinde die detektivische Methode des unterhaltenden Genres mit der in Philosophie, Dichtung und Wissenschaft, die als Detektions - Gebilde zu interpretieren seien. So wie es Freud in der Psychoanalyse um die Aufdeckung des subjektiv-falschen Bewusstseins zu tun sei, gehe es in der Gesellschaft um die Aufdeckung des objektiv Falschen. Beides bedarf der Detektion. Verbindendes Element zwischen Fiktion und philosophischen Entziffern, aus der die Bewegung, mithin das Prozessuale des Geschehens sich entwickele, sei die ,Rekonstruktion des Unerzählten'. Deshalb: „Etwas ist nicht geheuer, damit fängt das

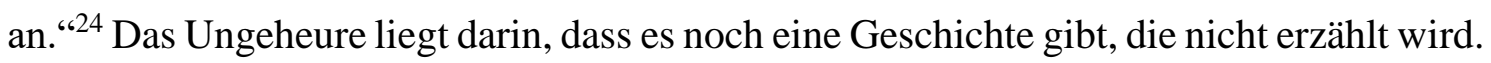
Der eigentliche Anfang, die Tat, die rekonstruiert werden soll, liegt vor dem Geschilderten. Um das Exemplarische dieser Erzählweise offen zu legen, zieht Bloch die Perspektive des Ödipus heran, denn auch für philosophische Theorien gilt, dass, „wenn auch gewiß kein ausphantasiertes Verbrechen, so doch ein Dunkel, ein Inkognito des Anfangs darin reflektiert worden ist. [...] und erst recht steht fest, dass kein Prozeß wäre, suchend, verändernd, möglicherweise gutmachend, wenn nicht etwas wäre, das nicht so sein sollte“. 25

Der Hinweis auf das Inkognito verweist nun vollends auf die Blochsche Philosophie selbst. Insbesondere bezieht er sich auf die Art und Weise, wie der Blick

\footnotetext{
${ }^{23}$ Vgl. G. Hausmranm a.a.O. Spatte 1382.

${ }^{24}$ Ernst Bloch. Philosophische Ansicht des Detektivromans. In: Ders.: Literarische Aufsätze. Frankfurt am Main 1984; S. 242.

${ }^{25}$ Ebd. S. $259 f$.
}

\begin{tabular}{|l|l|l|l|l|}
\hline Revista Dialectus & Ano 1 & n. 2 & Janeiro-Junho 2013 & p. 95-111 \\
\hline
\end{tabular}


auf die Welt im Werk von Bloch vollzogen wird. Diese Philosophie braucht die detektivische Analyse des Bestehenden und die Deutung der daraus abzuleitenden Tendenzen, weil sie die Veränderung des Gegebenen hin zum ,Reich der Freiheit' intendiert und deshalb die ,U-topoi als Orte im Prozeß des Sich-auf-den-Weg-machens' zu erhellen, gar zu besetzen beabsichtigt. Hier ist es der Philosoph, der beiträgt alle Zeichen zu entziffern, die auf jeweilige Entwicklungen und Tendenzen referieren. Es ist der Philosoph als Detektiv, der die Zeichen verstehen will, um in die Zukunft denken zu können.

Um Gemeinsamkeit und Differenz zwischen dem Verfahren des Detektivs und dem des Philosophen aufzeigen zu können, richte ich meinen Blick noch einmal auf die Charakterisierung des literarisch etablierten Detektivs. Auffällig sind seine Randposition und seine an das Leben von Künstlern erinnernden Gewohnheiten, zumindest wenn man das Klischee eines Künstlerlebens sich vor Augen führt. Im Blochschen Sinne gehören Detektive zu den utopischen Typen, die sich durch besondere Sensibilität auszeichnen. Diese Sensibilität äußert sich in dem Willen, die Welt zu ergründen. Dieser Wille wiederum macht sie zu produktiv Tätigen, die in der Welt nicht nur etwas finden, sondern sie durch das fabelnde Weiterdenken auch erfinden.

Doch brauchen sie einen Anlass, damit sie sich auf Spurensuche begeben. Auf den Detektiv sind es Verbrechen, die eine stimulierende Wirkung haben, ihn also zum Tätigwerden antreiben. Die Tat bewirkt, dass sich der Detektiv auf das konzentriert, was nicht stimmt. Sie befreien ihn zumeist aus einem als eintönig empfundenen Alltag, in dem er leicht zu Depressionen neigt und wecken den Drang, das scheinbar Dunkle zu lichten. Für den Verlauf der Geschichte bedeutet das, dass Spannung erst durch die Aufnahme der Spurensuche sich entwickelt. Volker Friedrich erklärt die Besonderheit des Detektivs durch seinen Hang zur Melancholie. ${ }^{26}$ Dieser habe seine Ursache in der Erkenntnis der Sinnlosigkeit des Todes. Deutlich äußert sich diese Stimmung darin, dass der Detektiv nach der Lösung des Verbrechens nicht euphorisch sei, sondern in Tatenlosigkeit zurückfalle. Am Beispiel der Figur des Sherlock Holmes wird dies mit dessen Wissen begründet, das einzelne Verbrechen zwar aufgeklärt zu haben, nicht aber dazu beitragen zu können, grundlegende Strukturen, die das Verbrechen erst ermöglichen, zu ändern.

\footnotetext{
${ }^{26}$ Vgl. Volker Friedrich. „Der Detektiv als melancholische Figur“, in: Ders.: Melancholie als Haltung. Berlin 1991, S. 131-152.
}

\begin{tabular}{|l|l|l|l|l|}
\hline Revista Dialectus & Ano 1 & n. 2 & Janeiro-Junho 2013 & p. 95-111 \\
\hline
\end{tabular}


Der Detektiv schaltet sich erst nach der Tat in das Geschehen ein, er rekonstruiert in der Überzeugung, dass jedes Geheimnis zu entschlüsseln sei, wenn man nur den Schlüssel dazu finde. Dafür bedient er sich der Gesetze der Logik. Im Gegensatz zum Philosophen reflektiert er das Verstehen jedoch nicht. Er sucht keine generellen Regeln der Aufklärung, hat kein Handbuch, sondern konzentriert sich auf den einzelnen Fall. So sind es oft nicht seine Beobachtungen, die den Leser in Erstaunen versetzten. Was er preisgibt, sind nicht die einzelnen Glieder, die ihn zur Lösung führen, sondern die Schlussfolgerungen selbst.

Ein solches Vorgehen ist freilich problematisch, weckt nicht nur Begeisterung, sondern auch Misstrauen. Dieses Problem wird schon in Voltaires Erzählung Zadig behandelt. ${ }^{27}$ Zadig gehört zu den Vorläufern der Kriminalgeschichte und beruht in seinem Kern auf einer vom orientalischen in den europäischen Raum tradierten Geschichte. In der Erzählung setzt sich der Deuter der Spuren dem Verdacht aus, selbst der Schuldige zu sein, weil auch er nur sein Ergebnis präsentiert. Einerseits behauptet er, vollkommen fremd im Ort des Geschehens zu sein, andererseits kann er das vor seinem Eintreffen Geschehene detailliert schildern. Auch er zog Schlüsse aus Beobachtungen und gab nur seine Schlussfolgerungen preis. Diese wirken, wenn sie in

allen Details beschrieben werden, durchaus überzeugend und beinhalten doch ein Moment des Ratens, es handelt sich um die wahrscheinlichste Lösung. Der Detektiv

zieht Schlüsse, die am wahrscheinlichsten sind, die aber durchaus auch andere Interpretationen zulassen. Es handelt sich also Vermutungswissen. Es sind Möglichkeiten, die der Detektiv aus den von ihm zusammengetragenen Fakten ermittelt.

Der Philosoph Ernst Bloch hat nicht das Ziel andere durch seine Schlussfolgerungen in Erstaunen zu versetzen. Mit Hannah Arendt lässt sich sagen, ihn geht es um das Verstehen. Durch die Widersprüche in Gesellschaft und Natur zum Staunen gebracht, wird der Wunsch nach Erkenntnis, mithin nach einem Verstehenwollen geweckt. Deshalb überträgt er den Gedanken, dass da etwas herausgebracht werden muss, was in der Sache selbst liegt, begrifflich aber noch nicht gefasst und daher offen ist, auf die Zukunft. Es geht ihm um das Sagen des Unsagbaren, quasi das Gegenteil des Wittgensteinschen Diktums. Denn auch Bloch wird vom nicht Geheurem motiviert, hat aber einen anderen Blick, den er selbst als auf Zukunft gerichtet beschreibt: „Forschend Aufdeckendes ist freilich nur das eine, es geht aufs Woher.

${ }^{27}$ Zadig ou La Destinée ist eine philosophische Erzählung von Voltaire, die dieser 1747 verfasste.

\begin{tabular}{|l|l|l|l|l|} 
Revista Dialectus & Ano 1 & n. 2 & Janeiro-Junho 2013 & p. 95-111 \\
\hline
\end{tabular}


Forschend Heraufbildendes wäre das andere, es geht aufs Wohin." ${ }^{28}$ Damit gibt er den Spuren eine geschichtliche Perspektive. Er unterscheidet sich vom rekonstruierenden, auf Vergangenheit blickenden Melancholiker, da er die Frage nach Erkenntnis immer mit der nach einer möglichen Praxis verbindet, was nicht leugnet, dass er dabei geschult wird durch den Umgang mit Enttäuschungen. Bei ihm gibt es trotzdem die Möglichkeit, die Welt zu ändern. Bloch betont: „Trotz Widerstands ist und bleibt die Welt so erkennbar wie demgemäß veränderbar, gegen die Meinung eines banal entschiedenen Pessimismus wie aller Gegenwart von Optimismus, der konsumierend statt militant auftreten zu können glaubt. Nicht nur wir, sondern die Welt selber ist noch nicht zu Hause; aber Erkenntnis, am Fluß des Vorhandenen wie an dem Ruf: ,Desto schlimmer für die Tatsachen' orientiert, zum Zuhausesein tendierend, kann unbestechlich Helferin sein in einer schweren Geburt. Heimat, dies prozeßhaft Vermittelbare, doch Ausstehende, Unnachlässliche hat zuerst die letzthinnige Evidenz von Wahren an sich, in sich; zentral problemhaft wohnt Angelangtsein utopisch im Kern von guten Möglichkeiten, in der Treue dazu. “29

Treu an den Möglichkeiten festzuhalten, das noch Uneingelöste nicht zu vergessen ist das Fundament der Hoffnung. Hier wird erkennbar, dass es nicht nur das Gefallen am literarischen Genre ist, dass den Auslöser gab über die Methode zu reflektieren. Vielmehr ist dies ein in der Kunst enthaltener Vorschein. Da in Blochs Philosophie das Sein als ein Noch-Nicht-Sein und damit als ein im Prozess des Werdens befindliches begriffen wird, haben ästhetische Gestaltungen einen besonderen Stellenwert. Sie sind Indizien für das, was in der Welt noch nicht stimmt, was aber als Fragment vorhanden ist und Spuren hinterlässt. Deshalb gibt das Lesen von Spuren generelle Hinweise auf die Regeln der Interpretation des Weltprozesses. Die Hinwendung zur Ästhetik ist also eine in kritischer Absicht, sie wird geleitet durch das Interesse an einer hypothetisch möglichen vernünftigen Welt. Kunst verweist als Darstellung des ,Charakteristisch-Typischen' auf die tendenziell erkennbare Invariante in der Welt. Entsprechend der Methodik des Detektivs sucht Bloch bei der Auslegung von Kunstwerken nicht ideelle Verweise, sondern das Stoffliche. Dieses Stoffliche ist die zum Ausdruck gebrachte Spannung zwischen Sein und Sollen.

\footnotetext{
${ }^{28}$ Ernst Bloch. Philosophische Ansicht des Detektivromans. In: Ders.: Literarische Aufsätze. Frankfurt am Main 1984; S. 261.

${ }^{29}$ Ernst Bloch. Experimentum Mundi. Frankfurt am Main: 1975, S. 60.
}

\begin{tabular}{|l|l|l|l|l|} 
Revista Dialectus & Ano 1 & n. 2 & Janeiro-Junho 2013 & p. 95-111 \\
\hline
\end{tabular}


Der Begriff des ,zum Ausdruck bringen' ist von Bloch dynamisch gemeint. Ausdruck ist eine Kategorie des Prozesses, in dem sich die Wahrheit erst durch die Veränderungen im Geschichtsprozess zu erkennen gibt. Deshalb ist mit Ausdruck kein bloßes Ausdrucksmittel gemeint, hingegen eine Kategorie, die Offenheit vermittelt. Dargelegt werden die Qualitäten der Kategorie vorrangig in der Musiktheorie ${ }^{30}$, von eher vagen Erläuterungen in ,Geist der Utopie' ausgehend bis zu der in ,Prinzip Hoffnung' erörterten Erkenntnis, dass Ausdruck eine humane Kategorie sei. Einerseits wird durch die Kategorie angezeigt, dass Inhalt niemals eindeutig sein kann, da er sich im Zeitverlauf wandelt; andererseits verweist sie sowohl auf die im Kunstwerk manifest gewordene Produktivität als auch auf die des Rezipienten. Es geht auch hier um die Wechselwirkung zwischen objektiv Gegebenen und subjektiv Empfundenen. Am Beispiel einer Tonfolge zeigt Bloch, dass das Verständnis für die zum Ausdruck kommende Spannung nicht allein durch Einfühlung bestimmt wird, indes auch durch einen in der Musik vorhandenen objektiven Faktor. Dieser weist der Einfühlung den Weg und macht es dem Hörer möglich, den nächsten Ton zu antizipieren. Zwischen Kunstwerk und Rezipient entsteht so eine dialektische Beziehung. Allerdings ist es hier der Hörer, der die Methode des Detektivs anwendet. Er mutmaßt über die Intention des Werkes und orientiert sich im Bemühen um Verständnis sowohl an Indizien als auch an seinem grundlegenden Weltwissen.

Ausdruck bietet so die Möglichkeit, die Sprengkraft der Kunst, ihre über die Wirklichkeit hinausgehende Zielperspektive zu bezeichnen. Damit diese zum Ausdruck gebrachte Spannung zum praktischen Handeln werden kann, sucht Bloch mit Rekurs auf Kant nach den Gesetzmäßigkeiten, nach einer Regel, die in der Sache selbst liegt. Bei der Suche nach den erst Vorscheinenden wird die Regel zum Schlüssel, das Mögliche zu erkennen. „Diese Regel“, so schon Kant, ,,aber ist für ein Wesen, bei dem Vernunft nicht ganz allein der Bestimmungsgrund des Willens ist, ein Imperativ, d.i. eine Regel, die durch ein Sollen, welches die objektive Nötigung der Handlung ausdrückt, bezeichnet wird, und bedeutet, dass, wenn Vernunft den Willen gänzlich bestimmte, die Handlung unausbleiblich nach dieser Regel geschehen würde. “31 Bloch betont die Zukunftsdeutung des Sollens bei Kant, lehnt aber eine als grundsätzlich festgelegte Differenz zwischen Sein und Sollen ab. Für ihn ist die Regel ein

\footnotetext{
${ }^{30}$ Vgl. Francesca Vidal: Bloch, in: Stefan Lorenz Sorgner; Oliver Fürbeth, Hg.: Musik in der deutschen Philosophie. Eine Einführung. Stuttgart: 2003, S. 135-152. Engl. in: St. Sorgner; O. Fürbeth, Ed.: Music in German Philosophy: An Introduction. University of Chicago Press 2011.

${ }^{31}$ Immanuel Kant: Kritik der praktischen Vernunft, 1. Teil. 22.
}

\begin{tabular}{|l|l|l|l|l|} 
Revista Dialectus & Ano 1 & n. 2 & Janeiro-Junho 2013 & p. 95-111 \\
\hline
\end{tabular}


Versprechen auf ein Sollen, dessen Drängen auf Einlösung geht. Dieses Drängen fordert zur Auslegung der Spuren, und so wird Blochs detektivisches Verfahren eine ,objektivreale Hermeneutik', eine die Erkennen und Produzieren in ihrer Einheit sieht.

Mit Gérard Raulet lässt sich diese Form der Hermeneutik als subversiv betiteln, da hier der Hoffnung eine methodische Funktion zugeschrieben wird. ${ }^{32}$ Mit der Hoffnung ,als Antriebskraft der praktischen Kritik $^{33}$ deute der Philosoph Manifestationen lebensweltlicher Erfahrungen und bestimmt den Interpreten als im Prozess Involvierten. Es ist dies eine detektivische Spurensuche, die die Welt als Ganzes zum Objekt der Auslegung wählt. Entscheidend aber ist, dass der Prozesscharakter der Welt dabei zur Grundlage des Entzifferns erhoben wird. Überträgt man dies auf die Auslegung der Lebenswelt als Deuten von Spuren, heißt das, sie nicht als unmittelbar anzunehmen, sondern als Produkte und als Teil des gesellschaftlichen Prozesses. Dadurch wird das traditionelle Verstehenskonzept um das Moment der Kritik erweitert. Bloch arbeitet den Drang zum Anderswerden aus den Dingen selbst heraus und siedelt dabei den Interpreten nicht außerhalb des Geschehens an. Er stellt seine Fragen nicht aus einer Zielperspektive heraus an den Text, sondern ist wie der literarische Detektiv Teil der Handlung.

Enthüllung mit den Methoden des Detektivs in der Philosophie verlangt ein aktives Mitdenken kritischer Praxis, denn sie will das Wesentliche jenseits der Erscheinungen aufspüren und vorantreiben. Vertreter hierfür werden in Prinzip Hoffnung ,Grenzüberschreiter, Pioniere, utopische Typen' genannt. Für sie stehen Don Quichotte und Faust, aber genauso auch der literarische Detektiv, denn sie verdeutlichen die Herausforderung an das philosophische Handeln, Erkenntnis um der Praxis willen zu betreiben.

Für mein Verständnis von Wissenschaft ist dies nicht antiquiert, sondern muss zurück erobert werden gegen alle die meinen, die Dinge so nehmen zu können wie sie scheinen. Ein Handbuch lässt sich daraus nicht ableiten. Auffällig, dass auch im neusten Roman ,Handbuch für Detektive“ dies eine Chimäre bleibt. Hier ist jemand, der unfreiwillig zum Detektiv geworden ist, der sich gibt, als sei er im Grunde mit seinem Job als Schreiber in einer Detektivagentur zufrieden, doch immer wieder dem Trott zu entgehen trachtet und dann den berühmtesten Detektiv suchen muss. Das Verbrechen

\footnotetext{
${ }^{32}$ Gerard Raulet. ,Subversive Hermeneutik des ,Atheismus im Christentum', in: Burghart Schmidt, Hg.: Seminar: Zur Philosophie Ernst Blochs. Frankfurt am Main 1983, S. 50-74.

${ }^{33}$ Ebd. S. 54.
}

\begin{tabular}{|l|l|l|l|l|}
\hline Revista Dialectus & Ano 1 & n. 2 & Janeiro-Junho 2013 & p. 95-111 \\
\hline
\end{tabular}


hängt mit dem Verschwinden des Detektivs zusammen, das Unheil mit den Methoden der Agentur, die die Grenze zum Privaten vollends ignoriert, da sie sich der Träume der Menschen bemächtigt. Bis zu seiner Aufgabe, dieses Rätsel zu lösen, erlebt der Held Unwin die Welt nur über die Berichte, die er schreiben muss. Jetzt muss er die Distanz aufgeben und sich entwickeln, um eigenständig zu handeln. Einzige Hilfe besagtes Handbuch, verfasst von einem Schreiber nicht von einem Detektiv. Der Roman handelt dabei genau von dem von Negt diagnostizierten Problem unserer Zeit, das Verhältnis von Nähe und Distanz, hier dargelegt am Abhören der Träume der Menschen durch die Detektivagentur. Auch dieser neue Detektiv lässt sich mit einem Blochschen Diktum beschreiben: Ich bin. Aber ich habe mich nicht. Darum werden wir erst. Mir scheint dies, ein passendes Motto für jegliches wissenschaftliche Arbeiten sei es nun in Philosophie oder Kulturwissenschaft. 